\title{
Persistent Mullerian Duct Syndrome
}

National Cancer Institute

\section{Source}

National Cancer Institute. Persistent Mullerian Duct Syndrome. NCI Thesaurus. Code C120188.

The presence of Mullerian duct-derived structures in a phenotypically male individual. 